\title{
Surface Freezing and a Two-Step Pathway of the Isotropic-Smectic Phase Transition in Colloidal Rods
}

\author{
Zvonimir Dogic* \\ IFF/Weiche Materie, Forschungszentrum Jülich, Jülich, D-52425 Germany
}

(Received 9 June 2003; published 16 October 2003)

\begin{abstract}
We study the kinetics of the isotropic-smectic phase transition in a colloidal rod/polymer mixture by visualizing individual smectic layers. First, we show that the bulk isotropic-smectic phase transition is preceded by a surface freezing transition in which a quasi-two-dimensional smectic phase wets the isotropic-nematic interface. Next, we identify a two-step kinetic pathway for the formation of a bulk smectic phase. In the first step a metastable isotropic-nematic interface is formed. This interface is wetted by the surface-induced smectic phase. In the subsequent step, smectic layers nucleate at this surface phase and grow into the isotropic bulk phase.
\end{abstract}

DOI: 10.1103/PhysRevLett.91.165701

PACS numbers: 64.75.+g, 64.70.Md, 82.70.Dd

Colloids with hard core repulsive interactions are often studied due to the simplicity and generality of their intermolecular potential. As a result of these studies, the equilibrium phase diagram of hard rods and spheres is well understood at the present time [1-4]. However, much less is known about the kinetic pathways of phase transitions in these systems [5,6]. Direct visualization of colloids in a system undergoing phase transition has provided a powerful tool to study general aspects of phase transition kinetics $[7,8]$. In this paper we study the kinetics of the isotropic-smectic phase transition by directly visualizing individual smectic layers in a phase separating sample. As a model system of colloidal rods we use a monodisperse suspension of the $f d$ virus [9]. We elucidate a kinetic pathway of unexpected complexity. The existence of surface freezing and a metastable isotropiccholesteric phase transition is discovered and their influence on the kinetic pathway is discussed. Because the behavior of the $f d /$ Dextran mixture is determined by steric interactions and since all molecules including low molecular weight thermotropics have a steric core, the results reported in this paper are likely to be quite general. In addition, our results might be pertinent to understanding the dynamics of amphiphilic membranes [10], 2D smectic systems [11] and surface freezing and wetting transitions [12-14].

It has been known for a long time that surface freezing/ melting can dramatically alter the nucleation rate and the kinetic pathway of a phase transition. On one hand, most substances exhibit surface melting. In this case a liquid surface wets the crystalline bulk phase. It follows that crystals melt from the surface inwards and therefore it is difficult to prepare a superheated metastable solid $[15,16]$. On the other hand, surface freezing is observed in very few systems, most notably thermotropic liquid crystals, alkanes and surfactant mesophases [12,13,17]. Upon supercooling these materials, the ordered phase nucleates at the frozen interface and propagates towards the bulk phase. Therefore, it is difficult to supercool liquids that exhibit surface freezing [18].

Another factor that can affect the nucleation rate of a transition is the presence of metastable phases [5,19]. For example, recent simulations predict that the free energy barrier for the formation of protein crystals is greatly reduced when a metastable gas-liquid phase transition is located in a vicinity of a stable liquid-solid phase boundary [5]. In this case the nucleation of protein crystals proceeds in two steps. In the first step a dense metastable droplet associated with the gas-liquid phase transition is formed, while in the subsequent step the protein crystal nucleates within this droplet. In this paper we show that both surface freezing and metastable phases are important for understanding the kinetics of the isotropicsmectic phase transition.

Bacteriophage $f d$ is a semiflexible virus with a contour length of $880 \mathrm{~nm}$, diameter of $7 \mathrm{~nm}$, and persistence length of $2200 \mathrm{~nm}$. It was prepared as previously described and dialyzed against a buffer of known ionic strength $(190 \mathrm{mM} \mathrm{NaCl}, 10 \mathrm{mM}$ Tris, $p \mathrm{H}=8.10)$. The phase diagram of the rod-polymer mixture was measured according to the published procedure [9]. All the samples are prepared in a metastable/unstable isotropic phase by shear melting any existing structure and are placed into rectangular capillaries (VitroCom, Mountain Lakes, NJ). Nucleation and growth of the order phase is observed with an optical microscope (Zeiss AxioPlan2) equipped with DIC (differential interference contrast) optics. All images are recorded with a cooled CCD (charge-coupled device) camera (AxioCam Zeiss).

At zero polymer concentration $f d$ is a good model system of hard rods and forms stable isotropic (I), cholesteric $(\mathrm{Ch})$, and smectic (S) phases with increasing concentration in agreement with theoretical predictions $[1,20]$. Equilibrium I-S phase transition is observed in a mixture of rodlike $f d$ viruses and nonadsorbing polymer Dextran. The phase diagram of this mixture is shown in 


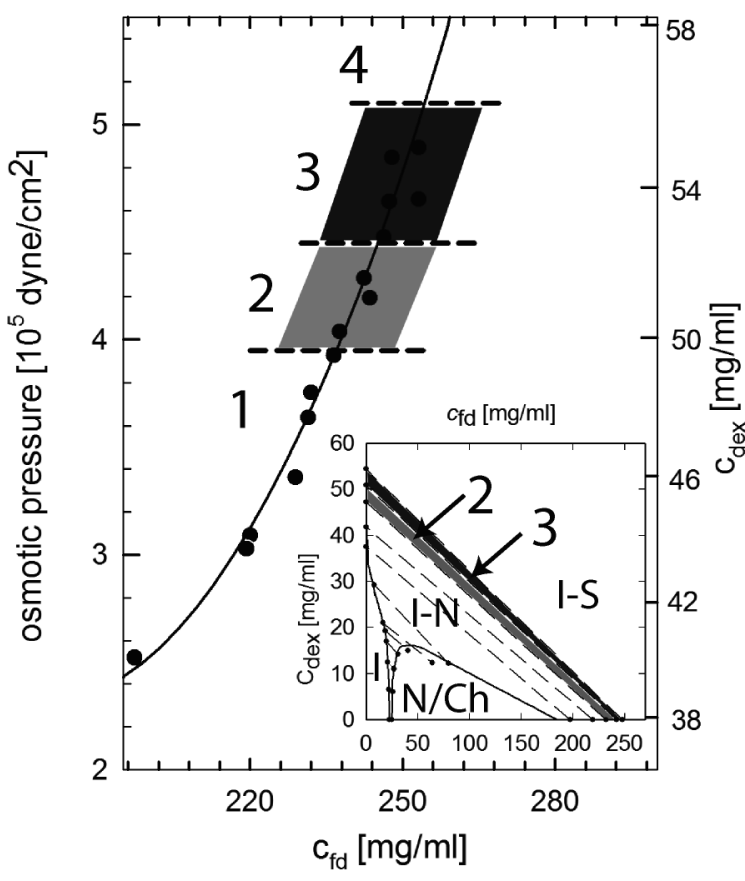

FIG. 1. The coexistence concentrations of an immiscible $f d /$ Dextran mixture. The $y$ axis shows the concentration and the osmotic pressure of Dextran in the isotropic phase that coexists with the rod-rich liquid crystalline phase (droplets). The rod concentration is shown on the $x$ axis. Numbers 1 through 4 indicate regions where different phase behaviors are observed. Images of the structures observed in these regions are shown in Fig. 2. A stable surface smectic phase wets the isotropic-nematic interface in region 2. Colloidal membranes are stable in region 3. Inset: The complete phase diagram of a $f d /$ Dextran mixture. Tie lines along which the phase separation proceeds are indicated by dashed lines. Regions of the isotropic-nematic (I-N) and isotropic-smectic (I-S) coexistence are indicated.

the inset of Fig. 1. Adding nonadsorbing polymer to $f d$ suspension produces effective attractive interactions between $f d$ rods [21]. The main consequence of this attractive potential on the phase behavior of a rodlike system is to widen the I-Ch coexistence concentrations with the polymer preferentially partitioning into the isotropic phase [22]. Since the interactions in the $f d$ /polymer mixtures are temperature independent, all phase transitions are entropically driven. In the first part of the paper we describe the equilibrium structures related to the surface freezing observed in region 2 of the phase diagram. In the second part of the paper we describe one of the kinetic pathways of phase separation observed in region 3.

At rod concentrations below $235 \mathrm{mg} / \mathrm{ml}$ (region 1 in Fig. 1 and inset), nematic droplets (tactoids) form in an isotropic background [Fig. 2(a)]. Polarization microscopy indicates that the configuration of rods in the nematic tactoid is as shown in Fig. 2(c). When confined to a small volume the cholesteric order is not able to develop, there-

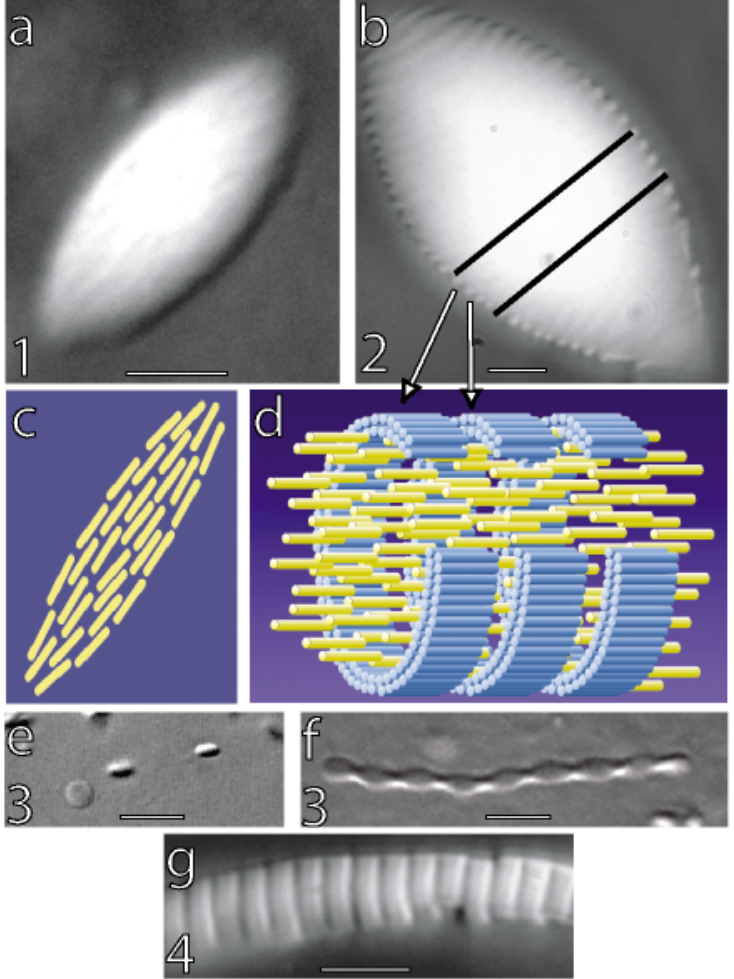

FIG. 2 (color online). Images and schematic representations of different structures observed in the $f d /$ Dextran mixture. (a) Anisotropic nematic droplet in the polymer rich background. Configuration of rods is shown in (c). (b) Nematic droplet with surface smectic phase. The image is formed by focusing on the midplane of the tactoids. The 3D structure is an object of revolution about the long axis. A schematic representation of a nematic droplet with surface-induced smectic phase is shown in (d). (e) Colloidal membranes which homogeneously nucleate from the isotropic phase. (f) A twisted ribbon which is identical to the colloidal membrane except that it is elongated along the twist directions. (g) Bulk isotropic-smectic phase coexistence. Scale bars indicate $3 \mu \mathrm{m}$.

fore we observe only unwound nematic phase within an individual tactoid. At higher rod concentrations (region 2 in Fig. 1) we observe droplets that have the same anisotropic shape. Microscopy indicates that the interior of these droplets is still nematic. However, each droplet has a corrugated isotropic-nematic (I-N) interface where the length of each ridge along the droplet's long axis is approximately one virus long. As the tactoids coalesce and increase in size, the surface corrugations are always confined to a narrow layer of well-defined thickness located at the I-N interface. This implies that the formation of corrugations is a purely surface effect. These observations lead us to conclude that there exists a surface-induced quasi-2D smectic phase that wets the I-N interface. The ridges observed at the interface are individual layers of the surface-induced smectic phase. A schematic representation of a section of a corrugated 
tactoid is shown in Fig. 2(d). The surface smectic phase is observed above an $f d$ concentration of $235 \mathrm{mg} / \mathrm{ml}$ while the bulk I-S phase transition (region 4) is observed at $255 \mathrm{mg} / \mathrm{ml}$.

After a few hours, the $f d$ /Dextran mixture prepared in region 2 completely phase separates with denser nematic tactoids coalescing and settling to the bottom of the sample. In this case a macroscopic I-N interface is formed. This makes it possible to focus on the interface and directly observe the surface-induced smectic phase (Fig. 3). We conclude our description of the system in region 2 by noting that there are no theoretical predictions of the surface-induced smectic phase in the rod/ polymer mixture. We expect that such a phase is a result of nonmonotonic density profiles across the I-N interface [23]. Additionally, in the $f d /$ polymer system rods in the surface frozen layer lie in the plane of the interface. This is in contrast to molecular systems which exhibit surface freezing where anisotropic molecules are either tilted or perpendicular to the interface $[12,13]$.

We now turn our attention to region 3 of the phase diagram. Right after mixing the sample, in addition to the formation of nematic droplets with a surface smectic, we observe a self-assembly of rods into disklike or ribbonlike structures [Figs. 2(e) and 2(f)]. The thickness of the disk corresponds to the length of a single rod. When viewed from above a disk shows no birefringence while from the side it shows maximum birefringence when oriented at $45^{\circ}$ with respect to the polarizer and analyzer [Fig. 4(d)]. Therefore, polarization microscopy shows that disks are composed of a monolayer of aligned rods in the smectic- $A$ configuration. We call these self-assembled disks colloidal membranes because of their similarity to amphiphilic membranes. Small homogeneously nucleated membranes [Fig. 2(e)] grow by coalescing laterally to form isolated membranes up to $40 \mu \mathrm{m}$ in diameter [Fig. 4(c)] [9]. This suggests that an isolated colloidal membrane and not a bulk smectic phase is the equilibrium structure in region 3. Polarization microscopy indicates

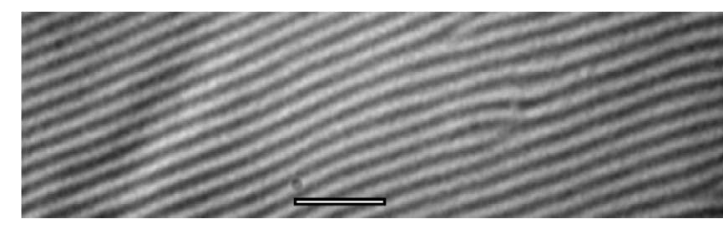

FIG. 3. Image of a macroscopically phase separated isotropic-nematic interface which exhibits surface freezing. The concentrations of the coexisting isotropic and nematic phases are $c_{f d}=242 \mathrm{mg} / \mathrm{ml}$ and $c_{\mathrm{dex}}=51.5 \mathrm{mg} / \mathrm{ml}$. The dense nematic phase is below the image plane while the isotropic phase is above the image. The thickness of the surfaceinduced smectic phase is a few hundred $\mathrm{nm}$. The surface structure shown here is identical to the surface of tactoids shown in Fig. 2(d). A pair of dislocation defects is clearly visible in the image. Scale bar indicates $5 \mu \mathrm{m}$. that twisted ribbons are identical to disks except that they have a twist along their long axes due to the chiral nature of $f d$ [24]. We expect that the free energy difference between these two morphologies is small and will examine their relative stability elsewhere.

Real space images enable us to study the kinetic pathway for the formation of colloidal membranes. They can either homogeneously nucleate from the metastable isotropic suspension or can heterogeneously nucleate at the surface-induced smectic phase [Figs. 4(a) and 4(b)]. A colloidal membrane nucleated at the interface grows into the isotropic phase either as a twisted ribbon or a flat disk. Over a period of a few days twisted ribbons nucleated at the I-N interface can reach lengths of several hundred microns. Fluorescence images indicate that there are no rods in the isotropic solution. Therefore colloidal membranes (ribbons) must elongate due to rods that diffuse from a metastable nematic phase through a surface smectic to a more stable colloidal membrane. The fact that
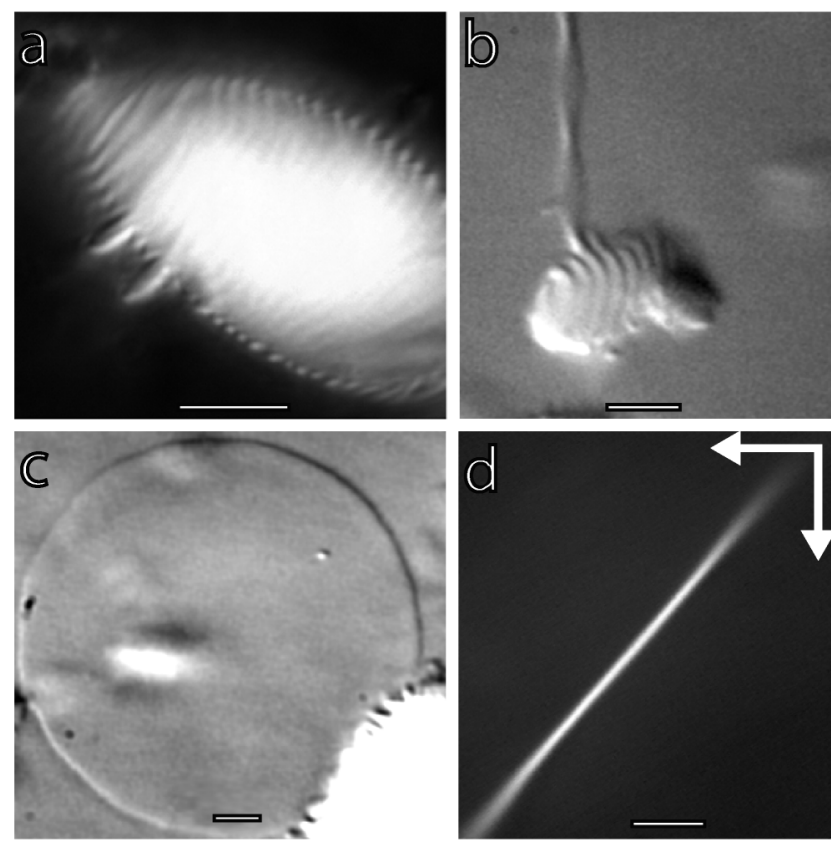

FIG. 4. Images of structures observed in region 3 of the phase diagram after the sample has been equilibrated for a few days. For images (a) and (b), $c_{f d}=254 \mathrm{mg} / \mathrm{ml}$ and $c_{\mathrm{dex}}=$ $53.5 \mathrm{mg} / \mathrm{ml}$, while, for images (c) and (d), $c_{\mathrm{dex}}=56 \mathrm{mg} / \mathrm{ml}$ and $c_{f d}$ is undetermined. (a) Nematic droplet with a surface frozen smectic phase. The surface smectic phase acts as a nucleation site for the formation of colloidal membranes. (b) The twisted smectic ribbon nucleates at the surface smectic phase and grows into the isotropic bulk phase. (c) DIC image of a large (35 $\mu \mathrm{m}$ diameter) isolated colloidal membrane in which the rods lie perpendicular to the image plane. Correspondingly the membrane shows no birefringence under crossed polarizers. (d) Polarization image of a colloidal membrane in which the rods lie in the plane of the image. Directions of the polarizer and the analyzer are indicated by white arrows. Scale bars indicate $5 \mu \mathrm{m}$. 
there is a transport of rods across the interface shows that the colloidal membranes are structures with lower free energy than the nematic phase or the bulk smectic phase. At lower degrees of supercooling we observe mostly heterogeneous surface-induced nucleation instead of homogeneous nucleation of colloidal membranes. This shows that a two-step kinetic pathway has a lower nucleation barrier for the formation of colloidal membranes. To summarize, the phase separation in region 3 of the phase diagram can proceed in two steps. In the first step, on a time scale of seconds to minutes, we observe the formation of nematic tactoids with a surface smectic phase identical to those observed in region 2. However, these tactoids are metastable. In the second slow step, on a time scale of hours to months, we observe the nucleation of colloidal membranes at the surface frozen smectic phase and their subsequent growth into the isotropic phase.

A few comments are in order regarding the structures observed in region 3. First, to our knowledge this is the first time that nonamhiphilic objects with very simple excluded volume interactions have been self-assembled into 2D membranelike [Fig. 4(c)] and 1D polymerlike structures [Fig. 2(f)] [25]. We speculate that these structures are stabilized by protrusionlike fluctuations [26]. Second, it seems plausible that isolated colloidal membranes observed in region 3 are highly swollen lamellar phases previously observed in mixtures of nematic $f d$ and hard spheres [4]. The swelling of the lamellar phase is predicted theoretically, but has yet to be observed in experiments. Third, as the osmotic pressure is increased there is a transition to region 4 in which small colloidal membranes irreversibly stack up on top of each other to form elongated filaments [Fig. 2(g)] [27]. The nature of the transition from isolated membranes to a smectic phase remains unexplored.

In conclusion, there are two important results that can be deduced from our experiments. The first surprising result is that a rod/polymer mixture exhibits surface freezing in which a quasi-2D smectic phase wets the I-N interface. This effect occurs at a rod concentration of $235 \mathrm{mg} / \mathrm{ml}$ while bulk I-S phase transition occurs at $255 \mathrm{mg} / \mathrm{ml}$. To our knowledge this is the first time that the surface freezing has been directly visualized in a system whose phase behavior is dominated by entropic repulsive interactions. The second result of this work is to demonstrate the relationship between the surface freezing and the bulk isotropic-smectic phase transition. A complex two-step kinetic pathway for the nucleation of the smectic phase out of the isotropic solution has been identified. In the first step a metastable nematic droplet with a surface frozen smectic phase nucleates in the isotropic solution. In the next step isolated monolayers (colloidal membranes) of smectic phase nucleate at the surface smectic phase and subsequently grow into the isotropic phase. Because of the simplicity and generality of the excluded volume interactions which dominate the phase behavior of the $f d$ /Dextran mixture, the present results should be relevant to a much wider class of systems than those studied here.

I wish to thank Seth Fraden, Gerhard Gompper, Arjun Yodh, Tom Lubensky, Daniel Chen, Peter Lang, and Pavlik Lettinga for useful discussions. I am particularly indebted to Jan Dhont for his hospitality at FZ-Juelich and the Alexander von Humboldt Foundation for financial support. Part of this work was done at Brandeis University where this research was supported by the NSF-DMR grant to Seth Fraden.

*Present address: Rowland Institute at Harvard, Cambridge, MA, USA.

[1] D. Frenkel, H. Lekkerkerker, and A. Stroobants, Nature (London) 332, 822 (1988)

[2] G. J. Vroege and H. N.W. Lekkerkerker, Rep. Prog. Phys. 55, 1241 (1992).

[3] P. N. Pusey and W. van Megen, Nature (London) 320, 340 (1986).

[4] M. Adams, Z. Dogic, S. L. Keller, and S. Fraden, Nature (London) 393, 349 (1998).

[5] P. R. ten Wolde and D. Frenkel, Science 277, 1975 (1997).

[6] J. Zhu et al., Nature (London) 387, 883 (1997).

[7] U. Gasser et al., Science 292, 258 (2001).

[8] V. J. Anderson and H. N.W. Lekkerkerker, Nature (London) 416, 811 (2002).

[9] Z. Dogic and S. Fraden, Philos. Trans. R. Soc. London, Ser. A 359, 997 (2001).

[10] R. Lipowsky, in Generic Interactions of Flexible Membranes, edited by R. Lipowsky and E. Sackmann (Elsevier, New York, 1995), pp. 521-602.

[11] C. Harrison et al., Science 290, 1558 (2000).

[12] X. Z. Wu et al., Phys. Rev. Lett. 70, 958 (1993).

[13] B. M. Ocko et al., Phys. Rev. Lett. 57, 94 (1986).

[14] D. Boon and D. Ross, Rep. Prog. Phys. 64, 1085 (2001).

[15] J. F. van der Veen, Surf. Sci. 435, 1 (1999).

[16] R.W. Cahn, Nature (London) 323, 668 (1986).

[17] P. Lang, J. Phys. Chem. B 103, 5100 (1999).

[18] E. Sloutskin et al., Phys. Rev. E 64, 031708 (2001).

[19] E. B. Sirota and A. B. Herhold, Science 283, 529 (1999).

[20] Z. Dogic and S. Fraden, Phys. Rev. Lett. 78, 2417 (1997).

[21] S. Asakura and F. Oosawa, J. Chem. Phys. 22, 1255 (1954).

[22] P. G. Bolhuis, A. Stroobants, D. Frenkel, and H. N.W. Lekkerkerker, J. Chem. Phys. 107, 1551 (1997).

[23] K. Shundyak and R. van Roij, Phys. Rev. Lett. 88, 205501 (2002).

[24] Z. Dogic and S. Fraden, Langmuir 16, 7820 (2000).

[25] A.V. Tkachenko, Phys. Rev. Lett. 89, 148303 (2002).

[26] J. Israelechvili, Intermolecular and Surface Forces (Academic Press, London, 1991), 2nd ed.

[27] D. Frenkel and T. Schilling, Phys. Rev. E 66, 041606 (2002). 